\title{
Cold QCD at finite isospin density: Confronting effective models with recent lattice data
}

\author{
Sidney S. Avancini®, ${ }^{1}$ Aritra Bandyopadhyay $\odot,{ }^{2}$ Dyana C. Duarte ${ }^{3},{ }^{3}$ and Ricardo L. S. Farias $\odot^{2}$ \\ ${ }^{1}$ Departamento de Física, Universidade Federal de Santa Catarina, \\ 88040-900 Florianópolis, Santa Catarina, Brazil \\ ${ }^{2}$ Departamento de Física, Universidade Federal de Santa Maria, \\ Santa Maria, Rio Grande do Sul 97105-900, Brazil \\ ${ }^{3}$ Departamento de Física, Instituto Tecnológico de Aeronáutica, \\ 12228-900 São José dos Campos, São Paulo, Brazil
}

(Received 7 August 2019; published 3 December 2019)

\begin{abstract}
We compute the QCD equation of state for zero temperature and finite isospin density within the Nambu-Jona-Lasinio model in the mean field approximation, motivated by the recently obtained lattice QCD results for a new class of compact stars: pion stars. We have considered both the commonly used traditional cutoff regularization scheme and the medium separation scheme, where in the latter purely vacuum contributions are separated in such a way that one is left with ultraviolet divergent momentum integrals depending only on vacuum quantities. We have also compared our results with the recent results from lattice QCD and chiral perturbation theory.
\end{abstract}

DOI: $10.1103 /$ PhysRevD.100.116002

\section{INTRODUCTION}

Quantum chromodynamics (QCD) is the fundamental theory of strong interactions. QCD has a remarkably rich phase structure with multiple facets which has been vividly explored over the years. Recently, with the imminent arrival of relativistic heavy ion collision (HIC) experiments in FAIR and NICA, physical systems at finite baryon densities such as neutron stars have become the ideal subject for scrutiny in the heavy ion community [1,2]. However, systems with finite baryon densities are not easy to deal with theoretically, since in this region of the QCD phase diagram, first-principle methods such as nonperturbative lattice calculations are not accessible due to the well-known fermion "sign problem" $[3,4]$. For a recent review about the progress of lattice QCD in dealing with the sign problem, see Ref. [5].

Aside from the baryon chemical potential $\mu_{B}=3\left(\mu_{u}+\right.$ $\left.\mu_{d}\right) / 2$ (for a two-flavor system), QCD at finite density can also be characterized by the isospin chemical potential $\mu_{I}=\left(\mu_{u}-\mu_{d}\right) / 2$. On the contrary to what happens at finite baryonic density, systems with finite isospin density do not suffer from the sign problem and hence are easily accessible to lattice QCD-based calculations. Initial results of

Published by the American Physical Society under the terms of the Creative Commons Attribution 4.0 International license. Further distribution of this work must maintain attribution to the author(s) and the published article's title, journal citation, and DOI. Funded by SCOAP ${ }^{3}$. lattice QCD at finite temperature and isospin density appeared in the early 2000s [6,7], and they were also investigated by other available techniques, such as chiral perturbation theory $(\chi \mathrm{PT})$ [8-17], hard thermal loop perturbation theory (HTLPt) [18], the Nambu-Jona-Lasinio (NJL) model [19-35] and its Polyakov-loop extended version PNJL [36,37], and the quark meson model (QMM) [38-41]. The results were also largely in qualitative agreement. However, all of the early lattice QCD calculations have been performed considering unphysical pion masses and/or an unphysical flavor content. Recently, this issue has been rectified by using an improved lattice action with staggered fermions at physical quark masses, and the modified lattice QCD results for finite isospin density are presented in Refs. [42-45].

In this work, we focus on a new type of compact stars, where the pion condensates are considered to be the dominant constituents of the core under the circumstance of vanishing neutron density. Moreover, this scenario is easily accessible through first-principle methods unlike the study of compact star interiors with high baryon densities. This novel scenario was first identified as pion stars in Ref. [11] and has recently been proposed through lattice QCD in Ref. [46].

Though pion stars can be described as a subset of boson stars [47-51], they are free from hypothetical beyondstandard-model contributions usually associated with boson stars, such as the QCD axion. Indeed, it can be proved in the framework of a dense neutrino gas that a Bose-Einstein condensate of positively charged pions can 
be formed [52]. Further exploration of the pion stars' equation of state (EOS) revealed their large mass and radius in comparison with neutron stars [46,53]. Recently, studies along similar lines have also been done within the chiral perturbation theory [54].

Though there are also possibilities of pion condensation in the early Universe driven by high lepton asymmetry $[52,55,56]$, in the current context we will consider the setting of compact stars with zero temperature. Further, the charged pion condensation requires accumulation of isospin charge at zero baryon density and zero strangeness. QCD, with $\mu_{I} \neq 0, \mu_{B}=\mu_{s}=T=0$, can be and is being realized well within lattice $\mathrm{QCD}$, and this new modified lattice result [46] in turn gives us the perfect platform for the consistency check of the effective models mimicking QCD, such as the NJL model. As emphasized earlier, QCD with finite isospin chemical potential has already been explored through the NJL model, albeit not in the light of the new improved lattice results. Additionally, the present study tries to rectify the regularization issues within the NJL model to deal with the ultraviolet (UV) divergent momentum integrals. In the traditional regularization scheme (TRS), commonly used in literature, the sharp UV cutoff $\Lambda$ usually cuts important degrees of freedom near the Fermi surface leading to incorrect results, especially in scales of the order of $\Lambda$, e.g., $\mu_{I} \sim \Lambda[57,58]$. On the other hand, the medium separation scheme (MSS), coined in Refs. [59,60], is based on a proper separation of medium effects from divergent integrals, originally having explicit medium dependence. This results in the disposal of all divergent integrals into the pure vacuum part-i.e., $\mu_{I}=0$ in the current context, as it should be. This scheme has already been successfully applied in the context of color superconductivity [57] and for quark matter with a chiral imbalance [59]. For a proper characterization of compact pion stars with high values of $\mu_{I}(\sim \Lambda)$, as we will be dealing with in this work, the role of the MSS becomes really important in this regard.

The paper is organized as follows: In Sec. II, we discuss the basic formalism of the two-flavor NJL model within both the TRS and the MSS. In Sec. III, we present our results obtained with the traditional regularization scheme and with the medium separation scheme; thermodynamic results are also presented and contrasted with other state-ofthe-art calculations. We conclude in Sec. IV by discussing the aftermath.

\section{FORMALISM}

In this section, we revisit the well-documented formalism for the two-flavor NJL model with finite isospin chemical potential [23-35]. We start with the partition function for the two-flavor NJL model at finite baryonic and isospin chemical potential, given by

$$
\begin{aligned}
Z_{\mathrm{NJL}}\left(T, \mu_{B}, \mu_{I}\right)= & \int[d \bar{\psi}][d \psi] \\
& \times \exp \left[\int_{0}^{\beta} d \tau \int d^{3} x\left(\mathcal{L}_{\mathrm{NJL}}+\bar{\psi} \hat{\mu} \gamma_{0} \psi\right)\right],
\end{aligned}
$$

where the quark chemical potential matrix in flavor space is

$$
\hat{\mu}=\left(\begin{array}{cc}
\mu_{u} & 0 \\
0 & \mu_{d}
\end{array}\right),
$$

and $\mu_{u, d}$ can be expressed in terms of the baryonic and the isospin chemical potential as

$$
\begin{aligned}
& \mu_{u}=\frac{\mu_{B}}{3}+\mu_{I}, \\
& \mu_{d}=\frac{\mu_{B}}{3}-\mu_{I},
\end{aligned}
$$

such that $\mu_{B} / 3=\left(\mu_{u}+\mu_{d}\right) / 2$ and $\mu_{I}=\left(\mu_{u}-\mu_{d}\right) / 2 . \mathcal{L}_{\mathrm{NJL}}$ appearing in Eq. (2.1) is the NJL Lagrangian considering scalar and pseudoscalar interactions, i.e.,

$$
\begin{aligned}
\mathcal{L}_{\mathrm{NJL}}= & \bar{\psi}(i \not \partial-m) \psi+G\left[(\bar{\psi} \psi)^{2}+\left(\bar{\psi} i \gamma_{5} \vec{\tau} \psi\right)^{2}\right] \\
= & \bar{\psi}(i \not \supset-m) \psi+G\left[(\bar{\psi} \psi)^{2}+\left(\bar{\psi} i \gamma_{5} \tau_{3} \psi\right)^{2}\right. \\
& \left.+2\left(\bar{\psi} i \gamma_{5} \tau_{+} \psi\right)\left(\bar{\psi} i \gamma_{5} \tau_{-} \psi\right)\right],
\end{aligned}
$$

where $\psi$ and $m$ represent the quark fields and their current mass, respectively, and $G$ is the scalar coupling constant of the model. $\tau$ 's are the generator matrices for the pseudoscalar interactions, which correspond to the pionic excitations $\pi_{1}, \pi_{2}, \pi_{3}$, or equivalently $\pi_{+}, \pi_{-}, \pi_{3}$, with $\tau_{ \pm}=\left(\tau_{1} \pm \tau_{2}\right) / \sqrt{2}$.

For finite isospin chemical potential, the isospin symmetry group $S U(2)$ explicitly breaks down to a subgroup $U(1)_{I_{3}}$, the third component of the isospin charge $\mathbf{I}_{3}$ being the generator [24]. So within the context of the mean field approximation, for nonzero $\mu_{I}$, one can consider the possibility of $\left\langle\bar{\psi} i \gamma_{5} \tau_{3} \psi\right\rangle=0$ as an ansatz, which further breaks the $U(1)_{I_{3}}$ symmetry. Now we can introduce the chiral condensate $\sigma=-2 G\langle\bar{\psi} \psi\rangle$ and pion condensates

$$
\begin{aligned}
& \sqrt{2} \pi_{+}=-2 \sqrt{2} G\left\langle\bar{\psi} i \gamma_{5} \tau_{+} \psi\right\rangle=\Delta e^{i \theta}, \\
& \sqrt{2} \pi_{-}=-2 \sqrt{2} G\left\langle\bar{\psi} i \gamma_{5} \tau_{-} \psi\right\rangle=\Delta e^{-i \theta},
\end{aligned}
$$

where the phase factor $\theta$ indicates the direction of the $U(1)_{I_{3}}$ symmetry breaking. Finally, for the present context of pion stars, we consider $\mu_{B}=0$, such that $\mu_{u}=-\mu_{d}=\mu_{I}$. Collecting all of this information, one can now obtain the thermodynamic potential within the mean field approximation as 
$\Omega_{\mathrm{NJL}}(\sigma, \Delta)=\frac{\sigma^{2}+\Delta^{2}}{4 G}-2 N_{c} \int_{\Lambda} \frac{d^{3} k}{(2 \pi)^{3}}\left[E_{k}^{+}+E_{k}^{-}\right]$,

where $E_{k}^{ \pm}=\sqrt{\left(E_{k} \pm \mu_{I}\right)^{2}+\Delta^{2}}$ with $E_{k}=\sqrt{k^{2}+M^{2}}$, $M=m+\sigma$, and the symbol $\int_{\Lambda}$ indicates integrals that need to be regularized.

The physical values of the condensates vis-à-vis the ground state at finite isospin chemical potential is determined by minimizing $\Omega_{\mathrm{NJL}}(\sigma, \Delta)$ with respect to the condensates $\sigma$ and $\Delta$-i.e., by solving the gap equations

$$
\left.\frac{\partial \Omega_{\mathrm{NJL}}}{\partial \sigma}\right|_{\sigma=\sigma_{m}}=\left.\frac{\partial \boldsymbol{\Omega}_{\mathrm{NJL}}}{\partial \Delta}\right|_{\Delta=\Delta_{m}}=0 .
$$

From these equations we obtain

$$
\begin{aligned}
& \sigma=4 G N_{c} M I_{\sigma}, \\
& \Delta=4 G N_{c} \Delta I_{\Delta},
\end{aligned}
$$

with the definitions

$$
\begin{gathered}
I_{\sigma}=\sum_{s= \pm 1} \int_{\Lambda} \frac{d^{3} k}{(2 \pi)^{3}} \frac{1}{E_{k}} \frac{E_{k}+s \mu_{I}}{\sqrt{\left(E_{k}+s \mu_{I}\right)^{2}+\Delta^{2}}} \\
I_{\Delta}=\sum_{s= \pm 1} \int_{\Lambda} \frac{d^{3} k}{(2 \pi)^{3}} \frac{1}{\sqrt{\left(E_{k}+s \mu_{I}\right)^{2}+\Delta^{2}}} .
\end{gathered}
$$

In the following subsections, we discuss in more detail different ways of regularizing these integrals. The thermodynamic quantities-i.e., the pressure, the isospin density and the energy density of the system, are then respectively given by

$$
\begin{gathered}
P_{\mathrm{NJL}}=-\Omega_{\mathrm{NJL}}\left(\sigma=\sigma_{m} ; \Delta=\Delta_{m}\right), \\
\left\langle n_{I}\right\rangle_{\mathrm{NJL}}=\frac{\partial P_{\mathrm{NJL}}}{\partial \mu_{I}}, \\
\varepsilon_{\mathrm{NJL}}=-P_{\mathrm{NJL}}+\mu_{I}\left\langle n_{I}\right\rangle_{\mathrm{NJL}} .
\end{gathered}
$$

Finally, the EOS within the two-flavor NJL model is given by the relation between $P_{\mathrm{NJL}}$ and $\varepsilon_{\mathrm{NJL}}$.

\section{A. TRS}

The TRS is the most common and used regularization scheme in the literature, as might be seen in some good reviews of the NJL model [61]. In this case, we just perform the integrations in Eqs. (2.8) and (2.9) up to a cutoff $\Lambda$, that becomes a model parameter. Therefore, the gap equations become $\sigma=4 G N_{c} M \int_{0}^{\Lambda} \frac{k^{2} d k}{2 \pi^{2}} \sum_{j= \pm 1} \frac{E_{k}+j \mu_{I}}{E_{k} \sqrt{\left(E_{k}+j \mu_{I}\right)^{2}+\Delta^{2}}}$,

$\Delta=4 G N_{c} \Delta \int_{0}^{\Lambda} \frac{k^{2} d k}{2 \pi^{2}} \sum_{j= \pm 1} \frac{1}{\sqrt{\left(E_{k}+j \mu_{I}\right)^{2}+\Delta^{2}}}$.

This same procedure is used in $\Omega_{\mathrm{NJL}}$, which becomes

$\Omega_{\mathrm{NJL}}^{\mathrm{TRS}}(\sigma, \Delta)=\frac{\sigma^{2}+\Delta^{2}}{4 G}-2 N_{c} \int_{0}^{\Lambda} \frac{k^{2} d k}{2 \pi^{2}}\left[E_{k}^{+}+E_{k}^{-}\right]$,

and also in the thermodynamic quantities. Specifically, the isospin density becomes

$\left\langle n_{I}\right\rangle_{\mathrm{NJL}}^{\mathrm{TRS}}=-2 N_{c} \int_{0}^{\Lambda} \frac{k^{2} d k}{2 \pi^{2}}\left[\frac{E_{k}-\mu_{I}}{E_{k}^{-}}-\frac{E_{k}+\mu_{I}}{E_{k}^{+}}\right]$.

\section{B. MSS}

Since NJL is nonrenormalizable, any physical quantity will depend on the scale of the model $\Lambda$. However, it is very important to keep in mind that cutoff-dependent medium terms, due to a naive regularization of the integrals, may lead to results completely different from the ones obtained with a more careful treatment of divergences. The MSS provides a tool to disentangle medium dependence from divergent contributions, so that only vacuum integrals need to be regularized. This scheme has been applied to the NJL model and successfully shows qualitative agreement with lattice simulations and more elaborated theories, as might be seen in Refs. [57,59,60].

The implementation of MSS starts by rewriting, for example, $I_{\Delta}$ given in Eq. (2.9) as

$I_{\Delta}=\frac{1}{\pi} \sum_{j= \pm 1} \int_{-\infty}^{+\infty} d x \int_{\Lambda} \frac{d^{3} k}{(2 \pi)^{3}} \frac{1}{x^{2}+\left(E_{k}+j \mu_{I}\right)^{2}+\Delta^{2}}$.

Using the identity

$$
\begin{aligned}
\frac{1}{x^{2}+} & \left(E_{k}+j \mu_{I}\right)^{2}+\Delta^{2} \\
= & \frac{1}{x^{2}+k^{2}+M_{0}^{2}} \\
& \quad+\frac{M_{0}^{2}-\Delta^{2}-\mu_{I}^{2}-M^{2}-2 j \mu_{I} E_{k}}{\left(x^{2}+k^{2}+M_{0}^{2}\right)\left[x^{2}+\left(E_{k}+j \mu_{I}\right)^{2}+\Delta^{2}\right]}
\end{aligned}
$$


(where $M_{0}$ is the vacuum mass, when $\mu_{I}=\Delta=0$ ) we obtain, after two iterations,

$$
\begin{aligned}
\sum_{j= \pm 1} & \frac{1}{x^{2}+\left(E_{k}+j \mu_{I}\right)^{2}+\Delta^{2}} \\
= & \frac{2}{x^{2}+k^{2}+M_{0}^{2}}+\frac{2 \mathcal{M}}{\left(x^{2}+k^{2}+M_{0}^{2}\right)^{2}} \\
& +\frac{2 \mathcal{M}^{2}+8 \mu_{I}^{2} E_{k}^{2}}{\left(x^{2}+k^{2}+M_{0}^{2}\right)^{3}} \\
& +\sum_{j= \pm 1} \frac{\left(\mathcal{M}-2 j \mu_{I} E_{k}\right)^{3}}{\left(x^{2}+k^{2}+M_{0}^{2}\right)^{3}\left[x^{2}+\left(E_{k}+j \mu_{I}\right)^{2}+\Delta^{2}\right]},
\end{aligned}
$$

where we have defined $\mathcal{M}=M_{0}^{2}-\Delta^{2}-\mu_{I}^{2}-M^{2}$. After some manipulations and performing the integration in $x$ indicated in Eq. (2.17), we obtain

$$
\begin{aligned}
I_{\Delta}^{\mathrm{MSS}}= & 2 I_{\mathrm{quad}}-\left(M^{2}-M_{0}^{2}+\Delta^{2}-2 \mu_{I}^{2}\right) I_{\log } \\
& +\left[\frac{3\left(\mathcal{M}^{2}+4 \mu_{I}^{2} M^{2}\right)}{4}-3 \mu_{I}^{2} M_{0}^{2}\right] I_{1}+2 I_{2}
\end{aligned}
$$

with the definitions

$$
\begin{gathered}
I_{\text {quad }}=\int \frac{d^{3} k}{(2 \pi)^{3}} \frac{1}{\sqrt{k^{2}+M_{0}^{2}}}, \\
I_{\log }=\int \frac{d^{3} k}{(2 \pi)^{3}} \frac{1}{\left(k^{2}+M_{0}^{2}\right)^{\frac{3}{2}}}, \\
I_{1}=\int \frac{d^{3} k}{(2 \pi)^{3}} \frac{1}{\left(k^{2}+M_{0}^{2}\right)^{\frac{5}{2}}}, \\
I_{2}=\frac{15}{32} \sum_{j= \pm 1} \int \frac{d^{3} k}{(2 \pi)^{3}} \int_{0}^{1} d t(1-t)^{2} \\
\times \frac{\left(\mathcal{M}-2 j \mu_{I} E_{k}\right)^{3}}{\left[\left(2 j \mu_{I} E_{k}-\mathcal{M}\right) t+k^{2}+M_{0}^{2}\right]^{\frac{7}{2}}},
\end{gathered}
$$

where, in the last line of the equation above we have used the Feynman parametrization

$\frac{1}{A_{1}^{n} A_{2}^{m}}=\frac{\Gamma(n+m)}{\Gamma(n) \Gamma(m)} \int_{0}^{1} d t \frac{t^{n-1}(1-t)^{m-1}}{\left[A_{1} t+A_{2}(1-t)\right]^{n+m}}$.

Using similar steps, one may write

$$
\begin{aligned}
I_{\sigma}^{\mathrm{MSS}}= & 2 I_{\mathrm{quad}}-\left(M^{2}-M_{0}^{2}+\Delta^{2}\right) I_{\log }+I_{3} \\
& +3\left[\frac{\mathcal{M}^{2}}{4}+\mu_{I}^{2}\left(M^{2}-M_{0}^{2}-\mathcal{M}\right)\right] I_{1}+2 I_{2},
\end{aligned}
$$

with

$$
\begin{aligned}
I_{3}= & \frac{15}{16} \sum_{j= \pm 1} \int \frac{d^{3} k}{(2 \pi)^{3}} \int_{0}^{\infty}(1-t)^{2} d t \\
& \times \frac{1}{E_{k}} \frac{j \mu_{I}\left(\mathcal{M}-2 j \mu_{I} E_{k}\right)^{3}}{\left[\left(k^{2}+M_{0}^{2}\right) t+\left(E_{k}+j \mu_{I}\right)^{2}+\Delta^{2}\right]^{\frac{7}{2}}} .
\end{aligned}
$$

Using the MSS, the expression for the normalized thermodynamic potential becomes

$$
\begin{aligned}
\Omega_{\mathrm{NJL}}^{\mathrm{MSS}}(\sigma, \Delta)= & \frac{\sigma^{2}+\Delta^{2}}{4 G} \\
& -2 N_{c}\left\{\tilde{\mathcal{M}} I_{\mathrm{quad}}-\frac{1}{4}\left(\tilde{\mathcal{M}}^{2}-4 \mu_{I}^{2} \Delta^{2}\right) I_{\log }\right. \\
& +\int \frac{d^{3} k}{(2 \pi)^{3}}\left[\frac{\tilde{\mathcal{M}}^{2}-4 \mu_{I}^{2} \Delta^{2}}{4 E_{k, 0}^{3}}-\frac{\tilde{\mathcal{M}}}{E_{k, 0}}\right. \\
& \left.\left.-2 E_{k, 0}+E_{k}^{+}+E_{k}^{-}\right]\right\},
\end{aligned}
$$

with the definitions $\tilde{\mathcal{M}}=\Delta^{2}+M^{2}-M_{0}^{2}$ and $E_{k, 0}=$ $\sqrt{k^{2}+M_{0}^{2}}$. To obtain the expression for the isospin density, we follow the same procedure used for the calculation of $I_{\Delta}$ and $I_{\sigma}$, but due to its different divergency structure we need to iterate the identity (2.18) once more. The final expression is

$$
\begin{aligned}
\left\langle n_{I}\right\rangle_{\mathrm{NJL}}^{\mathrm{MSS}}= & -2 N_{c}\left\{2 \mu_{I} \delta^{2} I_{\log }+3 \mu_{I}\left[\frac{\mathcal{M}^{2}}{4}+\mathcal{M}\left(M_{0}^{2}-M^{2}\right)\right.\right. \\
& \left.+M^{2} \mu_{I}^{2}+\frac{2 \mu_{I}^{2} M_{0}^{2}}{3}\right] I_{1} \\
& +2 \mu_{I} I_{2}-\frac{5 \mu_{I} M^{2}}{4}\left[3 \mathcal{M}^{2}+4 \mu_{I}^{2} M^{2}\right] I_{4} \\
& \left.+\frac{5 \mu_{I}}{4}\left(4 \mu_{I}^{2}\left(M_{0}^{2}-2 M^{2}\right)-3 \mathcal{M}^{2}\right) I_{5}+I_{6}\right\}
\end{aligned}
$$

with the remaining definitions

$$
\begin{aligned}
& I_{4}=\int \frac{d^{3} k}{(2 \pi)^{3}} \frac{1}{\left(k^{2}+M_{0}^{2}\right)^{\frac{7}{2}}}, \\
& I_{5}=\int \frac{d^{3} k}{(2 \pi)^{3}} \frac{k^{2}}{\left(k^{2}+M_{0}^{2}\right)^{\frac{7}{2}}},
\end{aligned}
$$




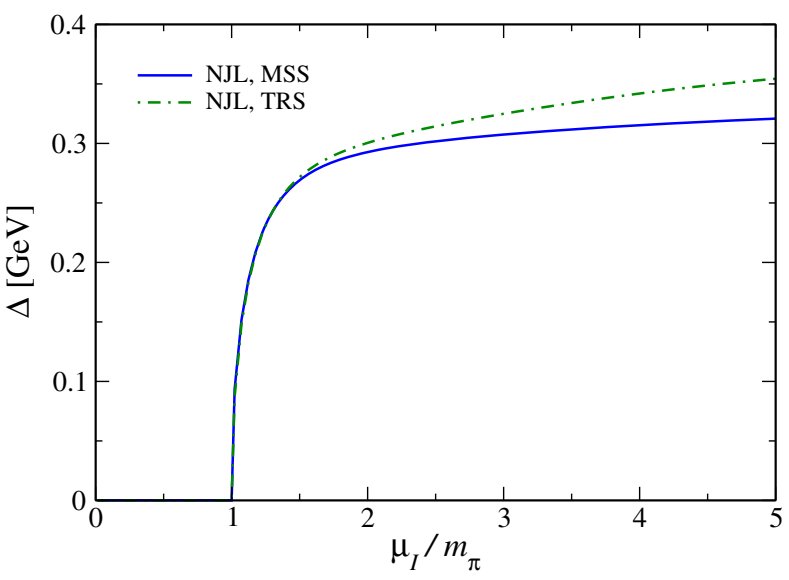

FIG. 1. Variation of the amplitude of the pion condensate $\Delta$ as a function of the normalized isospin chemical potential $\mu_{I} / m_{\pi}$, using both the TRS and the MSS.

$$
\begin{aligned}
I_{6}= & \frac{35}{32} \sum_{j= \pm 1} \int \frac{d^{3} k}{(2 \pi)^{3}} \int_{0}^{\infty} \frac{t^{3} d t}{\sqrt{1+t}} \\
& \times \frac{j E_{k}\left(\mathcal{M}-2 j \mu_{I} E_{k}\right)^{4}}{\left[\left(k^{2}+M_{0}^{2}\right) t+\left(E_{k}+j \mu_{I}\right)^{2}+\Delta^{2}\right]^{\frac{9}{2}}} .
\end{aligned}
$$

Note that integrals $I_{1}$ to $I_{6}$ are all finite, and must be performed up to infinite $k$. This is the fundamental difference between the TRS, where we cut the whole integral in the cutoff $\Lambda$, and the MSS, where all finite medium contributions are separated and performed for the whole momentum range.

\section{RESULTS}

The parameter set used for the purpose of the present study is $m=4.76 \mathrm{MeV}, \Lambda=659 \mathrm{MeV}$, and

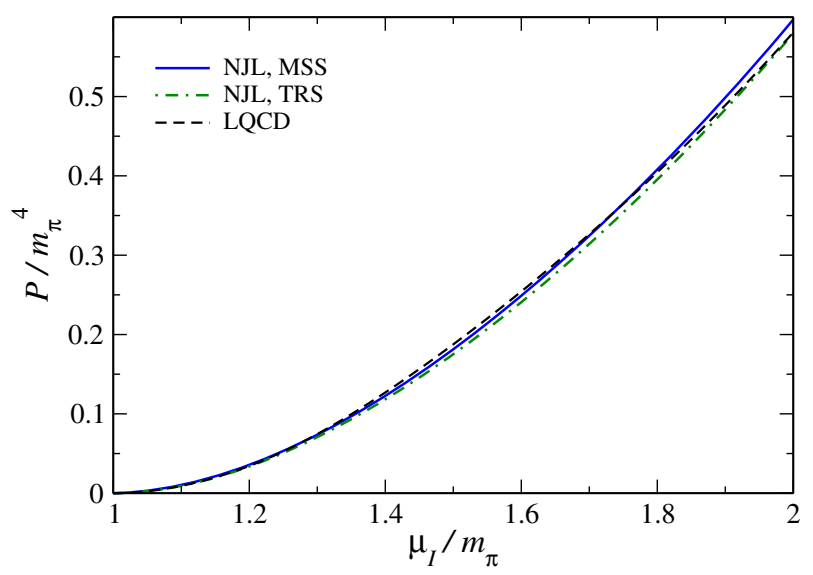

$G=4.78 \mathrm{GeV}^{-2}$, which we have obtained by fitting the same value of the pion mass as used by lattice QCD [62], i.e., $m_{\pi}=131.7 \mathrm{MeV}$, and other parameters as $f_{\pi}=$ $92.4 \mathrm{MeV}$ and $\langle\bar{\psi} \psi\rangle^{1 / 3}=-250 \mathrm{MeV}$. These values correspond to a vacuum mass $M_{0} \simeq 303.5 \mathrm{MeV}$.

Figure 1 shows the variation of the pion condensate $\Delta$ with $\mu_{I}$, scaled by the pion mass value. As might be seen from the plot, higher values of $\mu_{I}$ (starting from $\mu_{I} \sim 1.5 m_{\pi}$ ) draw the differences between the two regularization processes. Notice that the values of $\Delta$ are increasingly larger for the TRS than for the MSS when $\mu_{I}$ grows. At $\mu_{I} \sim \Lambda$ (i.e., $\mu_{I} \sim 5 m_{\pi}$ ), the difference between the TRS and MSS goes up to 30-35 MeV. This difference in $\Delta$ at higher values of $\mu_{I}$ also justifies the use of the medium separation scheme, especially since we are working at the zero-temperature limit.

In the following part of this section, we shall discuss our results for different relevant thermodynamic quantities within the two-flavor NJL model, comparing each one with the corresponding recent lattice QCD results [46] and chiral perturbation theory [54] results for both leading order (LO) and next-to-leading Order (NLO). It is important to mention that in the present study we are using datasets collected through private communications [63]. In the $\chi \mathrm{PT}$ results used in this study, the authors have used the Particle Data Group (PDG) value of the $f_{\pi}$, i.e., $\sqrt{2} f_{\pi}=130.2( \pm 1.7) \mathrm{MeV}$, and for the pion mass $m_{\pi}=135 \mathrm{MeV}$. Due to the uncertainty in the values of the low-energy constants $[54,63]$, the uncertainty for the $\chi$ PT-NLO results has also been presented. In Figs. 2, 3, and 4 , respectively, the variations of normalized pressure, isospin density, and energy density are shown with respect to the isospin chemical potential scaled by $m_{\pi}$. These plots have mainly focused on the region where $m_{\pi} \lesssim \mu_{I} \lesssim 2 m_{\pi}$ as the region of interest, throughout which lattice QCD data

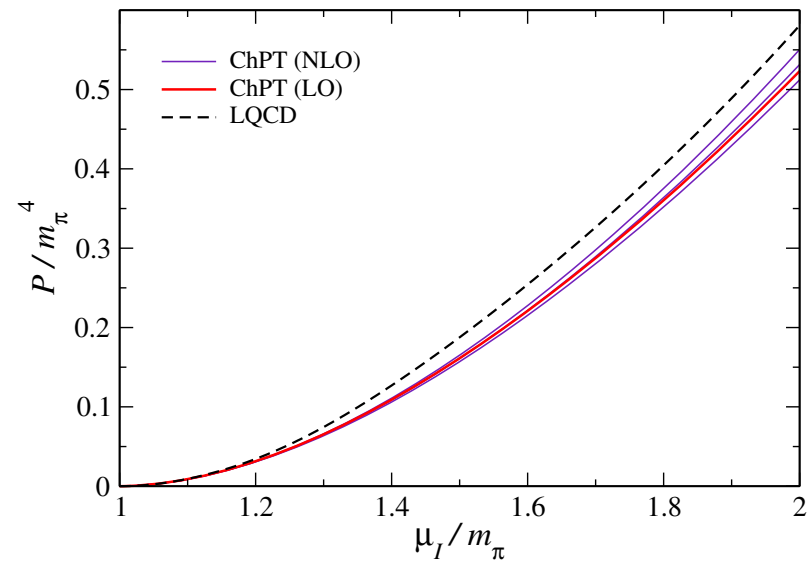

FIG. 2. Variations of the normalized pressure $\left(P / m_{\pi}^{4}\right)$ as a function of the normalized isospin chemical potential $\mu_{I} / m_{\pi}$. The LQCD results [46] have been compared with the behavior of the MSS and TRS within the NJL model (left panel) and with up-to-NLO results within $\chi$ PT [63] (right panel). Both the plots are specifically zoomed into the region of interest, up to the value of $\mu_{I}$ for which LQCD data are available. The three lines for $\chi$ PT-NLO depict the uncertainty in the result due to the uncertainty in the low-energy constants [54,63]. 

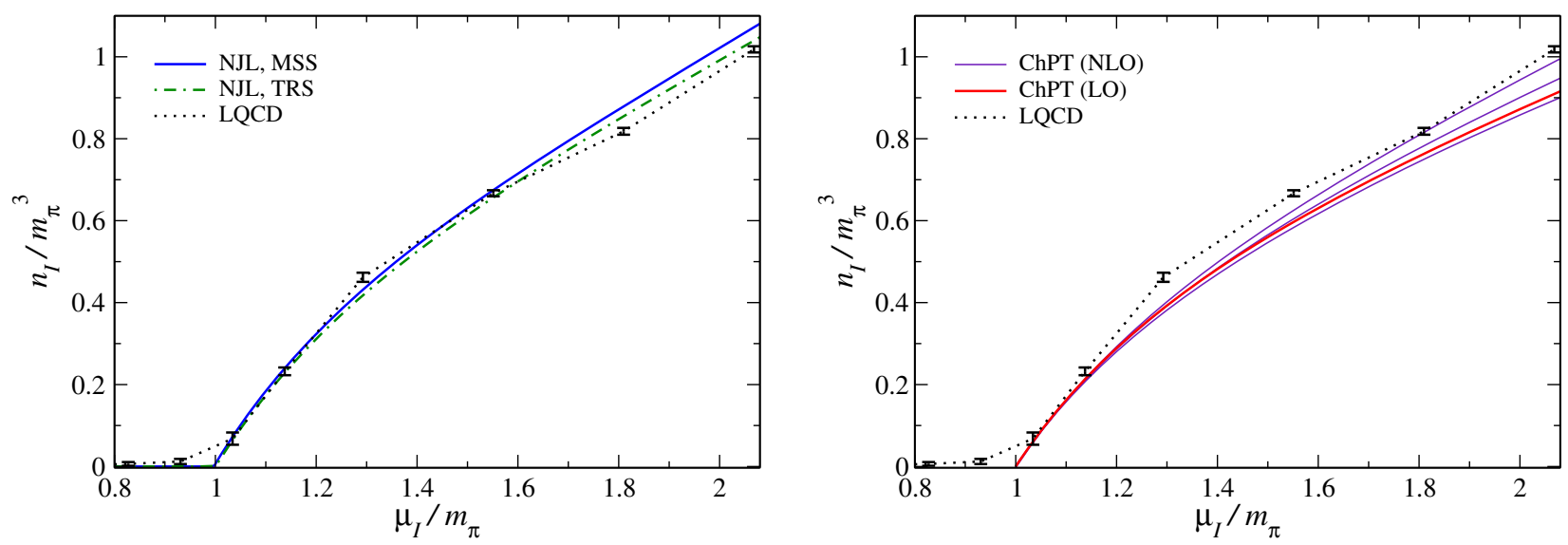

FIG. 3. Variations of the normalized isospin density $\left(n_{I} / m_{\pi}^{3}\right)$ as a function of the normalized isospin chemical potential $\mu_{I} / m_{\pi}$. The LQCD results [46] have been compared with the behavior of the MSS and TRS within the NJL model (left panel) and with up-to-NLO results within $\chi \mathrm{PT}$ [63] (right panel). The plots are specifically zoomed into the region of interest, up to the value of $\mu_{I}$ for which LQCD data are available. The three lines for $\chi$ PT-NLO depict the uncertainty in the result due to the uncertainty in the low-energy constants $[54,63]$. Unlike the other thermodynamic quantities, here relatively fewer lattice data points are shown with respective error bars. The dotted line represents the first-order interpolation of the latter.
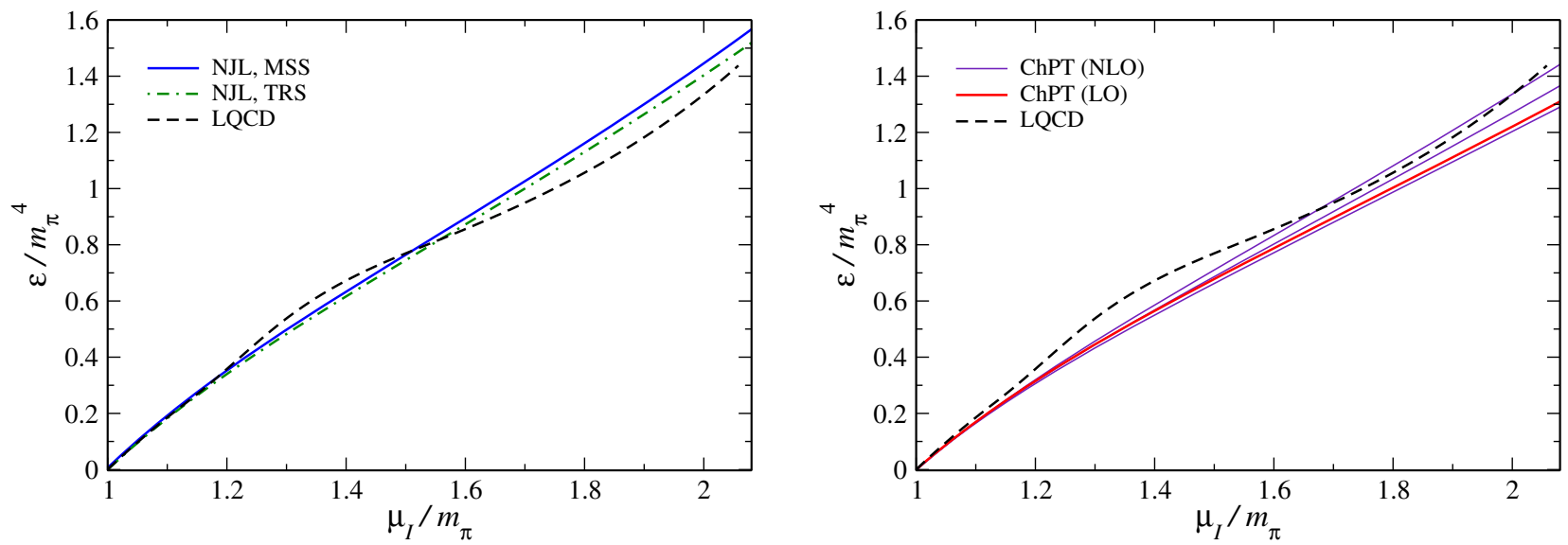

FIG. 4. Variations of the normalized energy density $\left(\varepsilon / m_{\pi}^{4}\right)$ as a function of the normalized isospin chemical potential $\mu_{I} / m_{\pi}$. The LQCD results [46] have been compared with the behavior of the MSS and TRS within the NJL model (left panel) and with up-toNLO results within $\chi$ PT [63] (right panel). The plots are specifically zoomed into the region of interest, up to the value of $\mu_{I}$ for which LQCD data are available. The three lines for $\chi$ PT-NLO depict the uncertainty in the result due to the uncertainty in the low-energy constants $[54,63]$.

were available. ${ }^{1}$ In this range of $\mu_{I}$, the difference in results for the TRS and MSS is relatively small, as is evident from the plots. Comparing NJL results, we can observe that the TRS has an infinitesimally better agreement with current LQCD than the MSS. LO and NLO results within $\chi$ PT have also been compared, among others. Figure 2 distinctively shows the comparability between the NJL and LQCD results, especially in comparison with $\chi \mathrm{PT}$ results up to NLO. Note that for the $\chi \mathrm{PT}$ datasets used here, the value of the pion mass used was taken as $135 \mathrm{MeV}$ (particle data group). Using instead a pion mass closer to the value

\footnotetext{
${ }^{1}$ In general, within lattice QCD calculations, the maximum value of $\mu_{I}$ is constrained by the value of the lattice spacing.
}

adopted by LQCD-i.e., $m_{\pi}=131 \pm 3 \mathrm{MeV}$ and $\sqrt{2} f_{\pi}=$ $128 \pm 3 \mathrm{MeV}$, as it is made in the published version of Ref. [54] - the agreement between LQCD and $\chi$ PT has been improved. Figures 3 and 4 show a typical behavior of LQCD data, which cross over the NJL TRS and MSS results around $\mu_{I} \sim 1.5 m_{\pi}$, though overall being largely in agreement. This crossover could be due to the current unavailability of a larger number of lattice data for isospin density.

The normalized EOS is presented in Fig. 5, where we can notice the reflection of the behavior of Figs. 3 and 4 regarding the comparability of NJL and LQCD results. As can be seen, within the limit of their uncertainties NLO $\chi$ PT results are in better agreement with the LQCD results for 

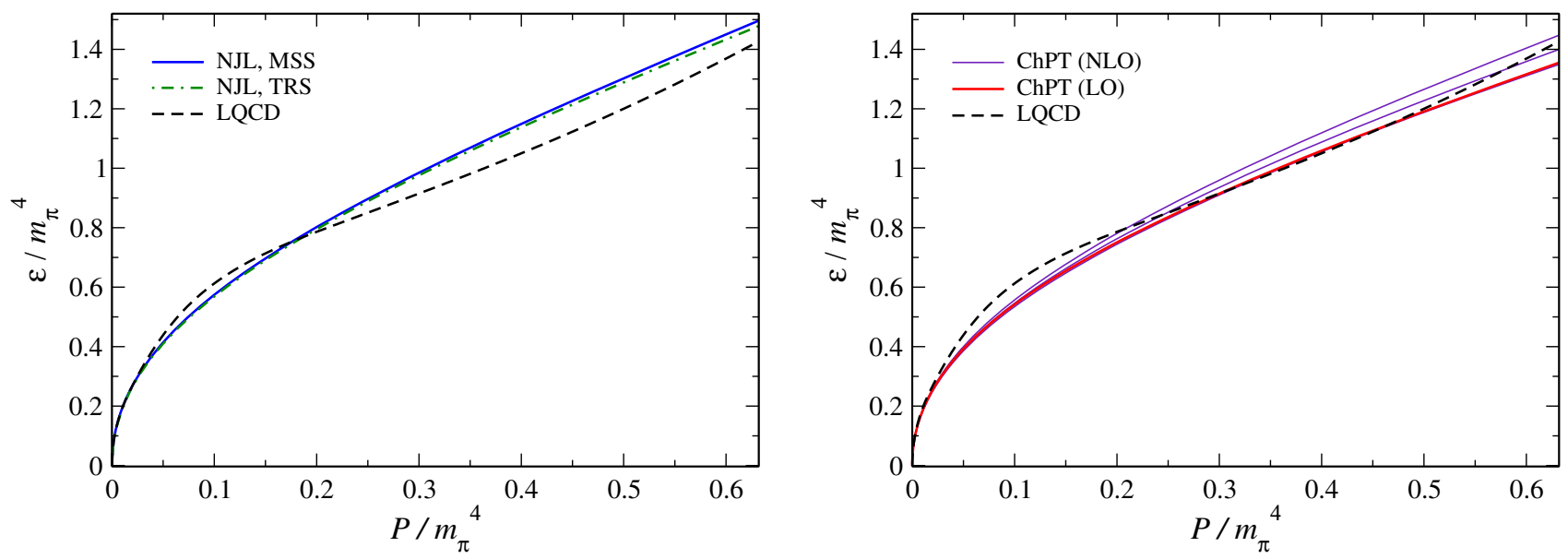

FIG. 5. Normalized equation of state. The LQCD results [46] have been compared with the behavior of the MSS and TRS within the NJL model (left panel) and with up-to-NLO results within $\chi$ PT [63] (right panel). The plots are specifically zoomed into the region of interest, up to the value of $\mu_{I}$ for which LQCD data are available. The three lines for $\chi$ PT-NLO depict the uncertainty in the result due to the uncertainty in the low-energy constants $[54,63]$.
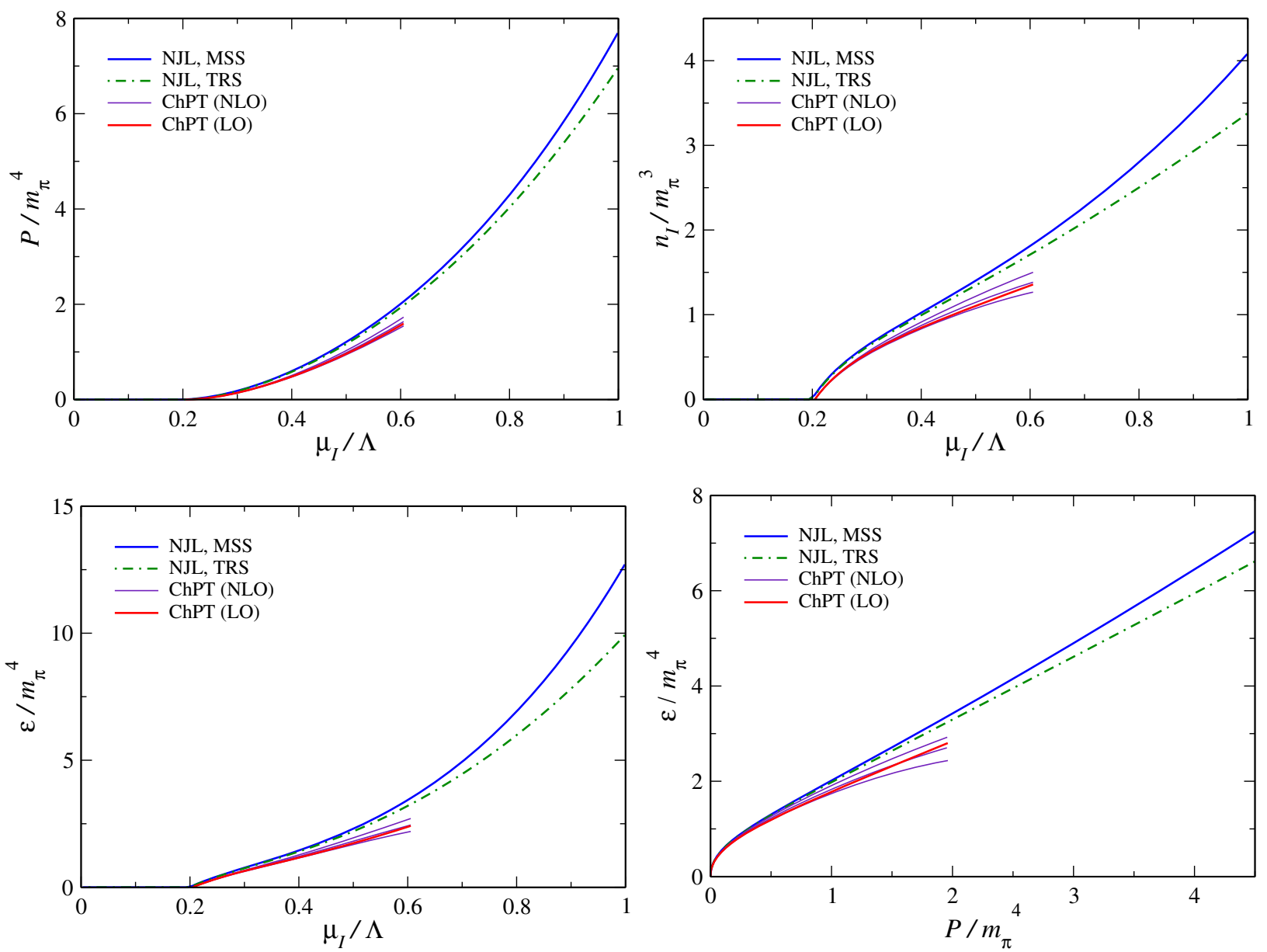

FIG. 6. Variations of the normalized pressure (upper-left panel), isospin density (upper-right panel), and energy density (lower-left panel) are shown as a function of isospin chemical potential scaled with the $3 \mathrm{D}$ momentum cutoff $\left(\mu_{I} / \Lambda\right)$ along with the normalized EOS (lower-right panel). This plot shows the different behaviors of the MSS and TRS within the NJL model over the full spectrum of $\mu_{I}$ up to $\Lambda$. $\chi$ PT results up to NLO have also been presented up to $\mu_{I}=0.6 \Lambda$. The three lines for $\chi \mathrm{PT}-\mathrm{NLO}$ depict the uncertainty in the result due to the uncertainty in the low-energy constants [63]. 
the region $P>0.2 m_{\pi}^{4}$, whereas NJL (TRS and MSS) results are in better agreement in the lower region of $P<0.2 m_{\pi}^{4}$.

Finally, in Fig. 6, we consider the full spectrum of $\mu_{I}$-i.e., $<0 \leq \mu_{I} \leq \Lambda$ - to emphasize the effect of the medium separation at higher values of $\mu_{I}$ on the normalized thermodynamic quantities $P_{\mathrm{NJL}},\left\langle n_{I}\right\rangle_{\mathrm{NJL}}$, and $\varepsilon_{\mathrm{NJL}}$, as well as the EOS. We interpret the parameter $\Lambda$ as the scale of the model, trusting in results restricted by $\Lambda$. In general, we use this $\Lambda$ as an upper limit for the other relevant variablese.g., temperature, external fields, chemical potentials, etc.and the same idea was applied for $\mu_{I}$ in this work. Though it is true that for $\mu_{I}=\Lambda$ the regime of validity of our model ends, we can see in Fig. 6 that the MSS results are different from the TRS even for $\mu_{I}<\Lambda$. We have also plotted $\chi \mathrm{PT}$ results up to NLO in Fig. 6, but only up to $\mu_{I}=0.6 \Lambda$ $\left(\sim 3 m_{\pi}\right)$. This is to emphasize the fact that those results cannot be trusted beyond $\mu_{I} \sim 3 m_{\pi}$ due to constraints on their validity [63].

\section{CONCLUSIONS}

In conclusion, we would like to emphasize the fact that both the TRS and the MSS regularization schemes within the NJL model show promising results in the front of thermodynamic quantities describing systems similar to pion stars, being largely in agreement with the LQCD results. For regions with higher values of $\mu_{I}$, where LQCD results are not available, we have predicted the pressure, isospin density, energy density, and EOS within both the TRS and the MSS, highlighting the fact that the MSS is more reliable in those regions due to its unique way of separating vacuum divergent effects from medium terms. In comparison with other effective theory results-i.e., $\chi \mathrm{PT}$ - our results within the mean-field NJL model show a better agreement with LQCD results, which prompts us to further investigate the phase diagram for the region with finite $\mu_{B}$ and $\mu_{I}$ which is inaccessible by LQCD due to the sign problem. Also, as mentioned in Sec. I, the possibility of pion condensation in light of the early Universe dictates further exploration in the $T-\mu_{I}$ plane of the QCD phase diagram. Furthermore, $\chi \mathrm{PT}$ calculations for $S U(3)$ at finite isospin have also appeared very recently in Ref. [64], which shows excellent agreement with lattice data for small values of $\mu_{I}$. Works in these directions within the NJL model are in progress.

\section{ACKNOWLEDGMENTS}

This work was partially supported by Conselho Nacional de Desenvolvimento Científico e Tecnológico (CNPq) under Grants No. 304758/2017-5 (R. L. S. F.) and No. 6484/2016-1 (S. S. A.), and as a part of the project INCT-FNA (Instituto Nacional de Ciência e TecnologiaFísica Nuclear e Aplicações) No. 464898/2014-5 (S. S. A.), Coordenacão de Aperfeiçoamento de Pessoal de Nível Superior (CAPES) (A. B.) and Fundação de Amparo à Pesquisa do Estado de São Paulo (FAPESP) under Grant No. 2017/26111-4 (D. C. D.).

Note added.-Recently, we learned that a partially overlapping study was done by Zhen-Yan Lu, Cheng-Jun Xia, and Marco Ruggieri [65].
[1] K. Fukushima and T. Hatsuda, Rep. Prog. Phys. 74, 014001 (2011).

[2] M. G. Alford, A. Schmitt, K. Rajagopal, and T. Schäfer, Rev. Mod. Phys. 80, 1455 (2008).

[3] F. Karsch, Lect. Notes Phys. 583, 209 (2002).

[4] S. Muroya, A. Nakamura, C. Nonaka, and T. Takaishi, Prog. Theor. Phys. 110, 615 (2003).

[5] P. F. Bedaque, EPJ Web Conf. 175, 01020 (2018).

[6] J. B. Kogut and D. K. Sinclair, Phys. Rev. D 66, 034505 (2002).

[7] J. B. Kogut and D. K. Sinclair, Phys. Rev. D 66, 014508 (2002).

[8] D. T. Son and M. A. Stephanov, Phys. Rev. Lett. 86, 592 (2001).

[9] D. T. Son and M. A. Stephanov, Yad. Fiz. 64, 899 (2001) [Phys. At. Nucl. 64, 834 (2001)].

[10] L. Lepori and M. Mannarelli, Phys. Rev. D 99, 096011 (2019).
[11] S. Carignano, L. Lepori, A. Mammarella, M. Mannarelli, and G. Pagliaroli, Eur. Phys. J. A 53, 35 (2017).

[12] O. Janssen, M. Kieburg, K. Splittorff, J. J. M. Verbaarschot, and S. Zafeiropoulos, Phys. Rev. D 93, 094502 (2016).

[13] T. D. Cohen and S. Sen, Nucl. Phys. A942, 39 (2015).

[14] E. S. Fraga, L. F. Palhares, and C. Villavicencio, Phys. Rev. D 79, 014021 (2009).

[15] M. Loewe and C. Villavicencio, Phys. Rev. D 67, 074034 (2003).

[16] M. Loewe and C. Villavicencio, Phys. Rev. D 71, 094001 (2005).

[17] K. Splittorff, D. T. Son, and M. A. Stephanov, Phys. Rev. D 64, 016003 (2001).

[18] J. O. Andersen, N. Haque, M. G. Mustafa, and M. Strickland, Phys. Rev. D 93, 054045 (2016).

[19] D. Ebert, T. G. Khunjua, and K. G. Klimenko, Phys. Rev. D 94, 116016 (2016). 
[20] T. G. Khunjua, K. G. Klimenko, R. N. Zhokhov, and V. C. Zhukovsky, Phys. Rev. D 95, 105010 (2017).

[21] T. G. Khunjua, K. G. Klimenko, and R. N. Zhokhov, Phys. Rev. D 98, 054030 (2018).

[22] T. G. Khunjua, K. G. Klimenko, and R. N. Zhokhov, Eur. Phys. J. C 79, 151 (2019).

[23] T. Xia, L. He, and P. Zhuang, Phys. Rev. D 88, 056013 (2013).

[24] C. F. Mu, L. He, and Y. X. Liu, Phys. Rev. D 82, 056006 (2010).

[25] H. Abuki, R. Anglani, R. Gatto, M. Pellicoro, and M. Ruggieri, Phys. Rev. D 79, 034032 (2009).

[26] J. O. Andersen and L. Kyllingstad, J. Phys. G 37, 015003 (2010).

[27] G. F. Sun, L. He, and P. Zhuang, Phys. Rev. D 75, 096004 (2007).

[28] D. Ebert and K. G. Klimenko, Eur. Phys. J. C 46, 771 (2006).

[29] D. Ebert and K. G. Klimenko, J. Phys. G 32, 599 (2006).

[30] L. He, M. Jin, and P. Zhuang, Phys. Rev. D 74, 036005 (2006).

[31] L. He, M. Jin, and P. Zhuang, Phys. Rev. D 71, 116001 (2005).

[32] L. He and P. Zhuang, Phys. Lett. B 615, 93 (2005).

[33] A. Barducci, R. Casalbuoni, G. Pettini, and L. Ravagli, Phys. Rev. D 69, 096004 (2004).

[34] D. Toublan and J. B. Kogut, Phys. Lett. B 564, 212 (2003).

[35] M. Frank, M. Buballa, and M. Oertel, Phys. Lett. B 562, 221 (2003).

[36] S. Mukherjee, M. G. Mustafa, and R. Ray, Phys. Rev. D 75, 094015 (2007).

[37] A. Bhattacharyya, S. K. Ghosh, A. Lahiri, S. Majumder, S. Raha, and R. Ray, Phys. Rev. C 89, 064905 (2014).

[38] P. Adhikari, J. O. Andersen, and P. Kneschke, Phys. Rev. D 98, 074016 (2018).

[39] R. Stiele, E. S. Fraga, and J. Schaffner-Bielich, Phys. Lett. B 729, 72 (2014).

[40] H. Ueda, T. Z. Nakano, A. Ohnishi, M. Ruggieri, and K. Sumiyoshi, Phys. Rev. D 88, 074006 (2013).

[41] K. Kamikado, N. Strodthoff, L. von Smekal, and J. Wambach, Phys. Lett. B 718, 1044 (2013).
[42] B. B. Brandt, G. Endrödi, and S. Schmalzbauer, Phys. Rev. D 97, 054514 (2018).

[43] B. B. Brandt, G. Endrödi, and S. Schmalzbauer, EPJ Web Conf. 175, 07020 (2018).

[44] B. B. Brandt and G. Endrödi, Proc. Sci., LATTICE2016 (2016) 039 [arXiv:1611.06758].

[45] B. B. Brandt, G. Endrödi, and S. Schmalzbauer, Proc. Sci., CONFINEMENT2018 (2018) 260 [arXiv:1811.06004].

[46] B. B. Brandt, G. Endrödi, E. S. Fraga, M. Hippert, J. Schaffner-Bielich, and S. Schmalzbauer, Phys. Rev. D 98, 094510 (2018).

[47] J. A. Wheeler, Phys. Rev. 97, 511 (1955).

[48] D. J. Kaup, Phys. Rev. 172, 1331 (1968).

[49] P. Jetzer, Phys. Rep. 220, 163 (1992).

[50] M. Colpi, S. L. Shapiro, and I. Wasserman, Phys. Rev. Lett. 57, 2485 (1986).

[51] S. L. Liebling and C. Palenzuela, Living Rev. Relativity 15, 6 (2012); 20, 5 (2017).

[52] H. Abuki, T. Brauner, and H. J. Warringa, Eur. Phys. J. C 64, 123 (2009).

[53] J. O. Andersen and P. Kneschke, arXiv:1807.08951.

[54] P. Adhikari, J. O. Andersen, and P. Kneschke, Eur. Phys. J. C 79, 874 (2019).

[55] D. J. Schwarz and M. Stuke, J. Cosmol. Astropart. Phys. 11 (2009) 025; 10 (2010) E01.

[56] M. M. Wygas, I. M. Oldengott, D. Bödeker, and D. J. Schwarz, Phys. Rev. Lett. 121, 201302 (2018).

[57] R. L. S. Farias, G. Dallabona, G. Krein, and O. A. Battistel, Phys. Rev. C 73, 018201 (2006).

[58] V. V. Braguta and A. Y. Kotov, Phys. Rev. D 93, 105025 (2016).

[59] R. L. S. Farias, D. C. Duarte, G. Krein, and R. O. Ramos, Phys. Rev. D 94, 074011 (2016).

[60] D. C. Duarte, R. L. S. Farias, and R. O. Ramos, Phys. Rev. D 99, 016005 (2019).

[61] S. P. Klevansky, Rev. Mod. Phys. 64, 649 (1992); T. Hatsuda and T. Kunihiro, Phys. Rep. 247, 221 (1994); M. Buballa, Phys. Rep. 407, 205 (2005).

[62] B. B. Brandt and G. Endrödi (private communication).

[63] P. Adhikari and J. O. Andersen (private communication).

[64] A. Prabal and J. O. Andersen, arXiv:1909.10575.

[65] Z. Y. Lu, C. J. Xia, and M. Ruggieri, arXiv:1907.11497. 